\title{
Immixtion du conflit nord-irlandais dans la vie politique française : le reportage des grèves de la faim en 1981 dans L'Humanité et Libération
}

\section{Karine Deslandes}

\section{(2) OpenEdition \\ Journals}

Édition électronique

URL : http://journals.openedition.org/etudesirlandaises/1770

DOI : $10.4000 /$ etudesirlandaises. 1770

ISSN : 2259-8863

Éditeur

Presses universitaires de Rennes

Édition imprimée

Date de publication : 30 juin 2010

Pagination : 55-67

ISSN : 0183-973X

\section{Référence électronique}

Karine Deslandes, «Immixtion du conflit nord-irlandais dans la vie politique française : le reportage des grèves de la faim en 1981 dans L'Humanité et Libération », Études irlandaises [En ligne],

35-1 | 2010, mis en ligne le 30 septembre 2012, consulté le 20 avril 2019. URL : http://

journals.openedition.org/etudesirlandaises/1770; DOI : 10.4000/etudesirlandaises.1770

Ce document a été généré automatiquement le 20 avril 2019.

(C) Presses universitaires de Rennes 


\title{
Immixtion du conflit nord-irlandais dans la vie politique française : le reportage des grèves de la faim en 1981 dans L'Humanité et Libération
}

\author{
Karine Deslandes
}

1 La grève de la faim des prisonniers républicains nord-irlandais, survenue en 1981 pendant la campagne pour les élections présidentielles en France, a fait couler beaucoup d'encre dans la presse française, qui, bien que condamnant à l'unanimité la politique du Premier ministre britannique de l'époque, Margaret Thatcher, a adopté envers les grévistes de la faim des positions bien différentes. En effet, si certains journaux, tels Le Monde ou La Croix, ont apporté un soutien limité aux prisonniers républicains, d'autres, comme Le Figaro, L'Express ou Réforme, un soutien quasi inexistant, les considérant comme «des terroristes ", il n'en est pas de même des quotidiens de gauche L'Humanité et Libération qui ont d'emblée soutenu les prisonniers de façon inconditionnelle, créant ainsi un clivage médiatique dans le paysage journalistique français. Il semblerait que les deux titres aient saisi l'occasion de rappeler, en période électorale, leur engagement aux côtés des partis de gauche et leur attachement aux droits de l'homme et aux valeurs démocratiques.

\section{Campagne de soutien aux grévistes de la faim}

2 Il faut d'abord mentionner que Libération et L'Humanité, contrairement aux autres publications françaises, avaient, depuis 1977, livré de nombreux reportages et enquêtes sur les conditions de détention des prisonniers républicains nord-irlandais ${ }^{1}$. C'est le quotidien communiste qui réagit le premier au début du jeûne de Bobby $\operatorname{Sands}^{2}$ et lui apporte immédiatement un soutien total. D'emblée, le journal appuie ouvertement cette forme d'action qu'il considère comme « un combat pour la dignité », « une lutte pour la liberté ». Il affirme que Bobby Sands "n'est pas un désespéré, mais un lutteur ${ }^{3}$ », il loue «une détermination et un courage peu communs ${ }^{4}$ » et conclut que «les jeunes 
prisonniers n'ont pas le choix. Ils n'ont plus d'autre moyen de défendre leur cause que de risquer leur vie, comme le combattant sur un champ de bataille ${ }^{5}$. L'Humanité et Libération dressent des portraits apologétiques des grévistes, les présentant comme des victimes du conflit: ils se sont engagés dans l'IRA parce qu'ils se sont retrouvés, à un moment donné, victimes de la discrimination, de l'injustice, de la violence britannique ou loyaliste ${ }^{6}$. Le journal communiste construit un véritable culte des héros, dont il commémore la mémoire à chaque nouveau décès par des martyrologes et des photos. Il publie également un appel à l'aide que les prisonniers républicains nord-irlandais auraient fait parvenir au Parti communiste français, signé par Tony Hughes (frère de Francis Hughes, le deuxième gréviste de la faim à avoir succombé) ${ }^{7}$. Le journal fait ensuite paraître la réponse du Comité de Défense des Libertés et des Droits de l'Homme, présidé par Georges Marchais, dans son édition du 13 mai 1981, soutenant les demandes des grévistes de la faim.

3 Libération, bien qu'absent pendant le jeûne de Bobby Sands (le quotidien n'a pas paru du 23 février au 11 mai 1981 inclus, étant confronté à une crise interne), publie dès sa reparution un article relatant rétrospectivement la grève de la faim de ce dernier ainsi qu'une enquête au titre explicite de la pensée de Sorj Chalandon : 'un soldat irlandais's. À la suite du décès de Joe McDonnell, le journaliste réitère son opinion :

Pour l'Irlande, la grève de la faim, c'est la dernière manière d'être une femme ou un homme libre, la dernière manière de refuser sa condition. Face à cela, Londres, Dublin, Paris ou Moscou peuvent tout tenter, cela ne servira à rien. Faites venir à leur chevet les curés, les phraseurs, les vedettes, les députés, les chefs de gouvernement, les Rois ou les Papes, cela non plus ne servira à rien. La solution et la fin de ces souffrances est dans l'acceptation sans condition de leurs revendications.

4 L'envoyé spécial du quotidien en Irlande du Nord couvre tous les événements de la perspective de la communauté catholique, mettant en avant ses réactions, ses émotions, ses points de vue ${ }^{10}$. Il décrit également les traditions funéraires de la communauté républicaine lors des obsèques, ou encore les appels à l'aide des parents des grévistes ${ }^{11}$. Sorj Chalandon livre des reportages parus dans la rubrique "événement» sur les stratégies des prisonniers, de la «bataille du vêtement civil » à la décision des grèves de la faim ${ }^{12}$. Il réfute l'argument invoqué par la presse britannique et par Le Monde $e^{13}$, selon lequel un ordre du commandement de l'IRA suffirait à faire cesser le mouvement. Il fait valoir que l'IRA/l'INLA, le Sinn Féin, ainsi que la communauté républicaine étaient opposés à ces jeûnes depuis le début, mais que :

L'Irlande nationaliste n'avait guère le choix. Elle devait, mise devant le fait accompli, soutenir ou condamner [...], alors toute la population républicaine d'Irlande du Nord se lançait dans la bataille. Ne soutenant pas la grève mais le gréviste, ne soutenant pas la mort mais la «bataille pour la vie ${ }^{14}$. "

5 Le journaliste justifie et soutient la réaction de la communauté nationaliste et celle des familles des grévistes qui refusent de donner l'ordre d'alimenter de force leurs enfants.

6 Fait particulièrement intéressant, Libération publie un court article provocateur signé Delfeil de Ton avouant : " J'ai beau me battre les flancs, pas moyen d'être ému par la mort de Bobby Sands. [...] Bobby Sands poussait la haine jusqu'à se haïr lui-même. Il s'est laissé mourir de faim. [...] Tu parles d'une connerie ${ }^{15}$. » Il y a une telle vague de colère parmi les lecteurs du quotidien que le journaliste doit démissionner ${ }^{16}$. Sorj Chalandon a d'ailleurs répondu à cet article en ces termes: "Quelle connerie la faim", pouvait-on lire il y a quelques semaines dans Libération. On pouvait aussi lire "quelle connerie les martyrs". Il 
n'y a pas de martyr en Irlande, il y a des hommes et des femmes qui tombent sous le coup des fusils ou celui de l'indifférence ${ }^{17}$."

7 Sur la question de savoir si les détenus doivent être considérés comme des prisonniers politiques ou de droit commun, les deux journaux reconnaissent le caractère politique de la lutte de l'IRA et l'INLA. L'Humanité répète inlassablement que "Bobby Sands, membre du Parlement, était un prisonnier politique, que les autres détenus républicains de Long Kesh sont des prisonniers politiques ${ }^{18}$ ", que leurs « revendications sont aussi modestes que légitimes ${ }^{19}$ ». Le quotidien communiste parle même de « la simplicité et de l'humanité des demandes formulées par les prisonniers ${ }^{20}$ ». À chaque nouveau décès d'un gréviste, il martèle que « les prisonniers de Long Kesh ne sont pas des criminels de droit commun, mais bien des détenus politiques qui se sacrifient pour leur cause ${ }^{21}$ ». Libération soutient également les revendications des prisonniers, qu'il énumère à la fin de chaque article. Les deux journaux justifient leur position en avançant plusieurs raisons: ils invoquent d'abord le fait que, dans une même prison, les détenus condamnés avant mars 1976 pour les mêmes délits bénéficient de ce statut privilégié2 ${ }^{2}$. Selon L'Humanité, cette situation rend le refus britannique « d'autant plus odieux », « d'autant plus insensé » et parle d'une « justice fondée sur deux poids et deux mesures ${ }^{23}$ ». Libération souligne qu'en République d'Irlande, les deux cents prisonniers républicains, «s'ils ne bénéficient pas d'un statut politique, peuvent vivre dans leurs vêtements civils 24 heures sur 24, ont le droit de libre association et la possibilité de ne pas faire de travail carcéral, ce que réclament les détenus des blocks $\mathrm{H}^{24}$ ». Serge July dénonce alors la « négation du caractère politique des actions et des motivations des militants de l'IRA, qui vient après une reconnaissance implicite jusqu'en 1976 ». Il livre ensuite le fond de sa pensée en faisant référence au nouveau gouvernement français de gauche :

Cette négation, les militants de l'IRA l'assument jusqu'au bout en souhaitant que de l'excès de négation surgisse une reconnaissance qui, quoiqu'on pense des méthodes de l'IRA, devrait aller de soi, non seulement en Grande-Bretagne mais dans toute l'Europe. En particulier en France où le nouveau régime fait redécouvrir aux Français le droit. Et ce droit est essentiel à toute démocratie ${ }^{25}$.

8 Le journaliste, qui soutient le gouvernement socialiste nouvellement élu en France, en attend clairement sinon une action en faveur des grévistes de la faim, du moins une reconnaissance de leur « combat ».

9 Les deux quotidiens invoquent également les lois d'exception en vigueur en Irlande du Nord et notamment les tribunaux sans jurés ${ }^{26}$. Le journal communiste ne cesse de proclamer que les détenus n'ont "jamais tué personne", que les chefs d'accusation retenus contre eux ne justifient pas les «peines exorbitantes». Parlant de la condamnation de Bobby Sands, il déclare: «il détenait une arme, c'est tout. Aucun témoin, rien dans l'acte d'accusation n'a dit qu'il s'était servi de cette arme. N'importe quel truand, dans les mêmes conditions, aurait écopé de quelques années de prison, probablement assorties du sursis ${ }^{27}$.» On retrouve les mêmes affirmations concernant les autres grévistes de la faim ${ }^{28}$. L'Humanité explique alors que « l'arbitraire colonial qui sévit en Irlande du Nord ne s'embarrasse pas de preuves. Etre membre de l'IRA suffit à justifier toutes les condamnations", et il va jusqu'à comparer les grévistes de la faim aux "résistants en France au temps de l'occupation nazie» et aux "Algériens qui participaient à la lutte de libération de leur pays ${ }^{29}$ ».

10 Une autre raison invoquée en faveur du statut de prisonnier politique est celle des résultats électoraux des grévistes de la faim. Les deux quotidiens ont constamment 
rappelé l'élection de Bobby Sands à Westminster et ont utilisé le terme « député » pour le désigner : ce n'est plus un simple prisonnier, mais un homme politique. L'Humanité met en exergue l'élection de Bobby Sands ${ }^{30}$, il est d'avis que le prisonnier «devrait siéger aux Communes » et dénonce le «non respect du suffrage universe ${ }^{31} »$. Il rappelle par ailleurs que cinq députés européens, parmi lesquels un membre du Parti communiste français, ont assisté à la cérémonie funèbre de Sands, y donnant ainsi un caractère politique officiel ${ }^{32}$. Lors des élections législatives dans la République d'Irlande, où neuf prisonniers, membres de l'IRA et de l'INLA, s'étaient portés candidats, deux furent élus députés au Dáil : Patrick Agnew et Kieran Doherty, respectivement pour les circonscriptions de Louth et Cavan Monaghan. Ces élections ont été suivies de près par les deux quotidiens français ${ }^{33}$ qui ne cesseront ensuite de rappeler que Kieran Doherty était député du Parlement irlandais.

11 Cette campagne de soutien aux prisonniers nord-irlandais s'accompagne dans les deux quotidiens d'une sévère condamnation de la politique britannique et de l'attitude de $\mathrm{M}^{\text {me }}$ Thatcher. L'Humanité est certainement le journal qui se distingue le plus par son engagement dans cette crise, par son ton combatif et son discours partisan. En effet, les grèves de la faim donnent au quotidien communiste l'occasion de mettre de nouveau en exergue le colonialisme, son cheval de bataille.

\section{« Intransigeance ", « inflexibilité », « mépris »: la méthode Thatcher}

À partir du 21 avril 1981, L'Humanité met en place une véritable campagne contre le Premier ministre britannique, participant ainsi à sa manière à la guerre de propagande. $\mathrm{Au}$ fur et à mesure que la crise s'aggrave, le journal se répand en invectives contre Mme Thatcher, seule responsable de cette tragédie. Il l'accuse d'abord de « calcul criminel qui bafoue les droits de l'homme et fait bon marché des vies humaines ${ }^{34}$ ", puis qualifie son refus catégorique d'accéder aux demandes des prisonniers de "provocation", d'«obstination insensée », d'« intransigeance glacée», ou encore d'«inflexibilité inhumaine » et de « détermination barbare ${ }^{35}$ ». Elle est accusée d' « [attiser] les feux de la violence ", de «[jeter] de l'huile sur le feu», de pratiquer «la politique du pire, une politique imbécile et sans issue ${ }^{36}$ ». À la mort de Bobby Sands, le journal se lance dans une véritable diatribe contre les autorités britanniques et ne parle plus que de «crime par non-assistance à personne en danger ». Il titre "Ils ont assassiné Bobby Sands » et déclare : «On l'a laissé mourir, dans des conditions telles que sa mort n'est rien d'autre qu'un assassinat. Ainsi l'exigeait la persistance d'une domination coloniale. Le lent supplice du député prisonnier jette l'opprobre sur le gouvernement de Mrs Thatcher ${ }^{37}$.»

À la suite de la mort de Francis Hughes, Martine Monod réitère : "Il s'agit d'un crime délibéré, prémédité. D'une série de meurtres dont la "Dame de fer" porte la responsabilité directe $^{38}$.» Puis Yves Moreau livre un article au titre évocateur «nous accusons» (titre qui n'est pas sans rappeler le célèbre article d'Emile Zola paru dans L'Aurore, «J'accuse », le 13 janvier 1898 lors de l'affaire Dreyfus), dans lequel le journaliste parle de «double crime où se manifeste toute la cruauté inhumaine du colonialisme ${ }^{39}$ ». À la suite du décès de Patsy O'Hara, le ton monte: «Mrs. Thatcher est responsable de toutes ces jeunes vies sacrifiées, du sang versé et des affrontements [...]. Les droits de l'homme, les droits de tout un peuple ne comptent pour rien aux yeux de cette femme si méchante ${ }^{40}$. » Les gros 
titres faisant la une sont d'ailleurs éloquents : « le second meurtre de Mme Thatcher » à la Une du 13 mai 1981, "Combien de morts $\mathrm{M}^{\mathrm{me}}$ Thatcher? " à la Une du 20 mai 1981, « dixième meurtre de $\mathrm{M}^{\text {me }}$ Thatcher » à la Une du 21 août 1981 avec les photos des 10 grévistes décédés. Il dissémine également de nombreuses caricatures satiriques du Premier ministre.

La visite surprise à Belfast du Premier ministre est considérée par le journal comme une « insulte à l'Irlande ", car « elle n'a pas eu un mot pour les victimes des militaires et des policiers, pas un mot pour les enfants tués dans la rue ${ }^{41} »$. Le quotidien relate également les différentes tentatives de médiation (délégation pontificale, commission Justice et Paix, la Croix-rouge internationale) et impute leur échec à « l'intransigeance du gouvernement de Londres ${ }^{42}$ ", contrairement au Monde et au Figaro qui avaient mis en avant le refus des prisonniers de rencontrer ces délégations sans représentant du Sinn Féin. Afin de bien faire comprendre aux lecteurs français son argument, L'Humanité rapproche les principes de $\mathrm{M}^{\mathrm{me}}$ Thatcher de « ceux qu'invoquaient les tortionnaires de l"“Algérie française" ${ }^{43}$ ".

Libération ne reste pas en marge dans la condamnation de la politique britannique. Dès sa reparution, il donne le ton avec pour titre en Une du journal « un deuxième mort pour Thatcher ", article dans lequel Sorj Chalandon dénonce «l'acharnement de Thatcher » et se demande si, « en plus d'une politique implacable, il ne faut pas voir une vengeance personnelle et épidermique comme si elle tenait à laver la mémoire de Lord Mountbatten et Airey Neave, son bras droit, tués l'un par l'IRA et l'autre par l'INLA ${ }^{44}$ ». Comme L'Humanité, à chaque nouveau décès d'un gréviste, le quotidien en fait porter l'entière responsabilité à Mme Thatcher. À la suite du décès de Joe McDonnell, Sorj Chalandon écrit : « des assassins en dame de fer, dame de mépris, dame de violence et Reine de mort ${ }^{45}$ ». Le 3 août 1981, à la suite du huitième décès, il titre en sa une «Irlande : Londres laisse mourir ", puis le 10 août, à la suite du neuvième décès : "Thatcher fait le mort », suivi de l'éditorial de Serge July, accusant le Premier ministre britannique de racisme :

Margaret Thatcher laisse mourir. Sans ciller, sans la moindre hésitation. Avec la plus totale froideur. La nature ferrugineuse de cette femme n'explique pas à elle seule ce comportement aux connotations totalitaires. Il faut à Margaret Thatcher, derrière elle, en elle, une tradition plusieurs fois séculaire de racisme anti-irlandais pour accueillir ainsi ces « exécutions-suicides » dans une prison britannique ${ }^{46}$.

Le journaliste qualifie ce comportement de «dernier reflet d'une attitude impérialiste: [...] c'est ce qui reste aux Britanniques lorsqu'on leur a tout pris, à commencer par leur grandeur industrielle, leurs hauts salaires et des pans entiers de leur fiertét ${ }^{47} »$.

Pour prouver l'inutilité de l'obstination de $\mathrm{M}^{\mathrm{me}}$ Thatcher, que certains journaux, comme Le Monde ou La Croix, expliquent par la peur de représailles du côté protestant, L'Humanité et Libération, soulignent le fait qu'aussi bien l'UDA (Association pour la défense de l'Ulster, principal groupe paramilitaire loyaliste) que l'Ordre d'Orange ont appelé le gouvernement britannique à faire des concessions dont pourraient également bénéficier les prisonniers loyalistes ${ }^{48}$. Il est également significatif que les deux journaux aient couvert les noces royales de Charles et Diana sur fond de grève de la faim. L'Humanité a publié à sa une du 29 juillet 1981 la photo des jeunes mariés à côté de celle des deux grévistes de la faim Kevin Lynch et Kieran Doherty avec pour légende : "Pendant que se déroulent aujourd'hui les fastes de la noce royale, les deux patriotes irlandais sont au seuil de la mort. La grande liesse officielle aurait pu être à Londres l'occasion d'un geste des autorités britanniques. " Libération a consacré deux articles au mariage princier: le premier, «les loyalistes pavoisent à Belfast», relate la joie et les préparations de 
l'événement dans les quartiers loyalistes et le second met en contraste «l'extase " à Londres et « la tristesse » à Belfast, « le visage rayonnant d'un prince et celui d'un homme de vingt-cinq ans qui va mourir ${ }^{49} »$.

18 Non seulement les deux quotidiens expriment leur soutien aux prisonniers et condamnent ouvertement la position du gouvernement britannique, mais ils ont également orchestré, notamment L'Humanité en tant qu'organe du Parti communiste français, une véritable campagne de mobilisation en France.

\section{Campagne de mobilisation en France}

19 L'Humanité ne cesse de marteler que « cela nous concerne, cela se passe dans notre monde dit libre, dans cette Communauté européenne », qu'« il faut les sauver », qu'« il est urgent d'intervenir ${ }^{50} »$. Il met en exergue l'action des communistes, les présentant ainsi comme les seuls défenseurs des droits de l'homme, ce qui n'est pas négligeable en temps de campagne électorale: il publie toutes les déclarations des députés européens communistes dans le cadre de la CE, les appels de la CGT et du Comité de Défense des Libertés et des Droits de l'Homme ${ }^{51}$, présidé par Georges Marchais et décrit toutes leurs démarches ${ }^{52}$. Le journal communiste organise la mobilisation de l'opinion publique française en appelant constamment les lecteurs à adresser des messages de protestations à l'ambassade de Grande-Bretagne à Paris, dont il prend bien soin de communiquer l'adresse à la fin de chaque article. Il publie également les appels des manifestations organisées par le PCF, la CGT ou le mouvement de la jeunesse communiste : le 6 mai 1981, le journal consacre sa une ainsi que toute la page six à appeler à la " manifestation ce soir à $18 \mathrm{~h} 30$ à Paris ", en fournit l'itinéraire et appelle à déposer des œillets rouges devant l'ambassade britannique ${ }^{53}$. Toutes les manifestations à Paris et en province sont largement couvertes par L'Humanité ainsi que tous les témoignages de solidarité et les actions entreprises par ses militants : retardement des trains, avions et car-ferries en partance pour Londres, occupation des locaux de la British Airways, constitution d'une garde d'honneur devant l'ambassade de Grande-Bretagne à Paris, organisation de multiples pétitions, la famille Sands invitée à la fête de l'humanités ${ }^{54}$. Le quotidien communiste a également soutenu la campagne du Secours populaire français d'aide aux familles des prisonniers par des appels aux dons, ainsi que la création à Paris le 14 août 1981 du Comité Solidarité-Irlande, constitué par des personnalités du monde politique, syndical, artistique, médiatique et universitaire : on note la présence des journalistes de L'Humanité, Yves Moreau et Martine Monod, de La Croix, Georges Baguet ainsi que du chanteur Jean Ferrat ${ }^{55}$.

Dans un contexte de tension électorale, L'Humanité en a profité pour constamment interpeller les dirigeants français et dénoncer leur «silence » : « On ne saurait estomper la responsabilité de ceux qui, chez nous, se sont tus alors que Bobby Sands agonisait. N'est-il pas honteux qu'aucun autre parti que le Parti communiste n'ait appelé à manifester ${ }^{56}$ ?» Ce n'est d'ailleurs pas innocent quand il déclare, à la veille du premier tour des élections présidentielles, dans un éditorial consacré à Bobby Sands : «C'est aussi ce [les assassinats politiques dans notre monde libre] qu'a dénoncé, au cours de sa campagne, le candidat du Parti communiste français. Pour les droits de l'homme chez nous, aussi bien qu'ailleurs dans le monde, le vote pour Georges Marchais sera dimanche le vote utile ${ }^{57}$.» Puis entre les deux tours de l'élection présidentielle, il dénonce : « Aucun des deux candidats [Valéry Giscard d'Estaing et François Mitterrand] n'a eu le moindre 
mot pour évoquer l'Irlande et le crime commis contre Bobby Sands. Comment ne pas s'en indigner et ne pas désavouer ce déshonorant mutisme ${ }^{58}$ ?» Il vilipende l'inaction de la CEE et accuse en particulier le Parlement européen d'« éclatante hypocrisie ", il en rapporte amplement les débats, décisions et "votes honteux ${ }^{59}$ ». Le quotidien communiste s'en prend également aux médias français : « Pourquoi faut-il que, parmi les journaux français à rayonnement national, nous soyons les seuls à formuler une telle accusation alors qu'il est indispensable de faire entendre à Londres toute l'indignation suscitée par de tels meurtres ${ }^{60}$ ?»

21 En dépit des affirmations de L'Humanité qui se dit le seul à défendre les prisonniers nordirlandais, Libération participe également à la campagne française de mobilisation en faveur des grévistes de la faim. Il évoque l'inaction de la CEE et du gouvernement français nouvellement élu, en se demandant "pourquoi l'Europe ne bouge pas, pourquoi le nouveau gouvernement de la France ne s'est pas encore manifesté alors que François Mitterrand avait envoyé un message de condoléances à la famille Sands ${ }^{61} »$. En remarquant qu' " autant l'émotion était intense en Europe après la mort de Sands, autant celles des autres Républicains emprisonnés n'ont pas soulevé la même colère ", il condamne "une sorte de banalisation qui s'est instaurée partout sauf en Irlande républicaine, où chaque nouveau décès est ressenti comme un assassinat britannique ${ }^{62}$ ». Il rappelle donc que les grévistes sont « morts à Long Kesh dans l'indifférence de l'opinion européenne $e^{63}$ ». Quand les prisonniers annoncent la cessation de la grève de la faim, Sorj Chalandon, dans un éditorial intitulé « une défaite collective ", s'en prend aux dirigeants européens et au nouveau président français :

Dès la mort de Bobby Sands, le doute s'était instauré, conforté par neuf autres victimes et par le silence de l'Europe. Car enfin, la victoire britannique n'a pu se faire que grâce aux regards détournés des autres pays, des autres dirigeants, des intellectuels et des abonnés aux droits de l'homme. Le 10 mai 1981, dans la nuit, je traversais Falls Road, à Belfast, couverte de barricades en feu. Une femme s'est approchée de moi et m'a embrassé. Une vieille Irlandaise comme le ghetto catholique en connaît des milliers : «Avec votre président socialiste, a-t-elle dit, nous avons la victoire assurée. Il va se battre pour les droits de l'homme, nous ne sommes plus seuls. » Dans l'émotion du moment, j'ai eu l'imbécillité de le croire. Il ne fallait pas, c'était idiot et criminel. Cette victoire de Thatcher est une victoire collective dans ce sens où elle a été rendue possible par le désintérêt collectif. Elle est aussi pour les droits de l'homme une défaite collective ${ }^{64}$.

Le journal a également consacré un article à la venue à Paris de la mère de Kieran Doherty alors en grève de la faim depuis 43 jours, et du père Faul, aumônier catholique de la prison de Maze. Il a retracé la conférence de presse donnée par ces derniers à l'initiative du Comité de défense des prisonniers politiques irlandais, appelant le Président Mitterrand «à intervenir vite ${ }^{65}$ ». Un mois après, à la suite du décès de Kieran Doherty, Sorj Chalandon a de nouveau publié un article, intitulé «En France: "Kieran comment?" ", dénonçant l'apathie des Français. Rappelant que pour Bobby Sands "Mitterrand, alors candidat à la présidence de la République, avait tenu en son nom et celui de sa femme à apporter ses condoléances à la famille du jeune Irlandais ", il se demande pourquoi il n'en est pas de même pour Kieran Doherty, lui aussi député, et dénonce « le grand silence du Parti socialiste ». Il regrette qu'à « ce jour, personne, à part trois parlementaires du Parti socialiste [qui étaient mandatés par la Ligue internationale des Droits de l'Homme et non par leur groupe parlementaire], n'[ait] fait le geste d'aller à Belfast, à une heure d'avion et quelques minutes de Paris ${ }^{66}$ ». Le 11 septembre 1981, le quotidien souligne la rencontre d'Owen Carron $^{67}$ et de Sean Sands (le frère du défunt 
gréviste) avec Lionel Jospin, alors Premier secrétaire du Parti socialiste français et mentionne à cette occasion: "Première démarche du PS en faveur des Irlandais depuis l'élection de François Mitterrand ${ }^{68}$. $»$ On retient que Libération a amplement soutenu la création et les actions du Comité de défense des prisonniers politiques irlandais à travers divers articles ${ }^{69}$. Il a également annoncé les diverses manifestations organisées par le PCF ou la CGT, suivi de la mention « y participer est le moins que nous puissions faire $\mathrm{e}^{70}$ ».

\section{Conclusion}

L'Humanité a couvert les grèves de la faim en Irlande du Nord de façon partisane et son reportage reflète plus son idéologie communiste que ce qui s'est réellement passé dans la province. Le journal, sur un ton combatif, dénonce l'impérialisme, ici britannique, la répression et défend les libertés et les droits de l'homme. Il semble que le quotidien se soit servi des événements en Irlande du Nord pour redorer l'image des communistes en sérieux déclin, les présentant comme les seuls capables de défendre les valeurs de la démocratie française. La position de Libération, qui défend également les prisonniers républicains nord-irlandais, apparait moins surprenante dans la mesure où le quotidien s'est toujours montré pro-républicain et a toujours considéré les membres de l'IRA provisoire comme « des combattants », « des soldats ». En revanche, L'Humanité avait, dès 1971, différencié les deux branches de l'IRA : alors qu'il soutenait de manière univoque la branche officielle de l'organisation, à idéologie marxiste, il condamnait ouvertement la branche provisoire qu'il considérait comme des «terroristes ». Il avait même mis en garde contre « ce que font malheureusement de trop nombreux commentateurs [français] de presse et de radio, prendre la partie pour le tout » et rappelait que « les "provisionals", dissidents de l'IRA, ne sont qu'une partie de l'IRA ${ }^{71} »$. Tout au long des années 1970, il a martelé que les "provos » pratiquaient le «terrorisme aveugle ». Il est donc étonnant qu'en 1981, il se lance dans cette campagne de soutien des prisonniers de l'IRA provisoire. Ce revirement fait tout simplement penser à un calcul politique en période électorale en France. De plus, après les grèves de la faim de 1981, contrairement à Libération qui, au fil des ans, a continuellement livré à ses lecteurs de nombreux reportages sur les événements en Irlande du Nord et notamment sur l'IRA provisoire et le Sinn Féin, se faisant en quelque sorte le «porte-parole » français du mouvement républicain irlandais, L'Humanité a montré un net désintérêt: il a considérablement réduit sa couverture des «Troubles » nord-irlandais et les nouvelles du conflit se sont d'un coup raréfiées pour les lecteurs communistes français.

\section{NOTES}

1. Voir entre autres Libération du 23 décembre 1977, «Cinq années dans les camps britanniques » et du 17 mai 1979, « Nus depuis trois ans sous les couvertures » par Sorj Chalandon et L'Humanité du 15 avril 1980, « Une guerre clandestine au flanc de l'Europe », par Henri Alleg. Dès 1976, les prisonniers républicains refusent d'endosser l'uniforme 
carcéral, pour protester contre la suppression du statut de prisonnier politique : ils vivent nus sous leur couverture et ne peuvent donc plus sortir de leur cellule. En mars 1978, pour protester contre les violences et humiliations auxquelles ils sont soumis, ils entament la grève de l'hygiène. La grève de la faim est le dernier recours qu'ils ont trouvé pour faire pression sur le gouvernement britannique de façon à obtenir le rétablissement du statut politique.

2. L'Humanité du 3 mars 1981, « Pour des conditions humaines de détention ».

3. L'Humanité du 4 mai, la Une et p. 7, par Yves Moreau.

4. L'Humanité du 2 mai, la une et p. 7, par Jean-Émile Vidal.

5. L'Humanité du 21 mai 1981, p. 7, par Jean-Émile Vidal.

6. L'Humanité du 6 mai 1981, du 15, 16, 23 mai 1981, du 9 juillet 1981 entre autres. Libération du 15 mai, du 23/24 mai 1981, du 9 juillet 1981, des 3, 4, 10 et 21 août 1981.

7. L'Humanité du 9 mai 1981, p. 6: «Lettre de la prison », par Tony Hughes (version anglaise et la traduction en français).

8. Libération du 15 mai 1981, p. 18-20.

9. Libération du 9 juillet 1981.

10. L'article du 13 mai 1981 décrit la mobilisation des ghettos catholiques dans l'attente de la mort de Francis Hughes et celui du 14 mai 1981 est intitulé « Hughes : la réaction du ghetto ».

11. Voir Libération du 23 juin 1981.

12. Libération du 25/26 juillet, p. 2-3 et du 4 août 1981, la Une et p. 3.

13. Le Monde du 5 août 1981, p. 5 par Claire Tréan.

14. Libération du 4 août 1981, p. 3 par Sorj Chalandon.

15. Libération du 13 mai 1981, dernière page, par Delfeil de Ton.

16. Voir Maurice Goldring, "The Northern Ireland Conflict and the French Left", in John Darby, Nicholas Dodge and A.C. Hepburn (eds.), Political Violence. Ireland in a Comparative Perspective, 1990, p.110. Cet article forme le chapitre 7 de l'ouvrage collectif: "The Northern Ireland Conflict in the French Press" et est une compilation de plusieurs parties: Paul Brennan ayant écrit sur Le Figaro, Richard Deutsch sur Le Monde, Elisabeth Gaudin sur la presse religieuse et Maurice Goldring sur les journaux de gauche. Cet article est la seule source secondaire publiée sur le reportage du conflit nord-irlandais par la presse française. Voir à ce sujet la thèse de doctorat de l'auteur «Étude de l'image médiatique du conflit nord-irlandais dans la presse française (1968-1998) », University of Ulster, 2009.

17. Libération du 9 juillet 1981, par Sorj Chalandon.

18. L'Humanité du 9 mai, p. 6, par Jean-Émile Vidal.

19. L'Humanité du 4 mai 1981, la Une et p. 7, par Yves Moreau.

20. L'Humanité du 14 mai 1981, p. 6, par Jean-Émile Vidal.

21. L'Humanité du 15 juin 1981, par Jean-Émile Vidal.

22. Un statut spécial, proche du statut de prisonnier politique, avait été accordé aux détenus des organisations paramilitaires en juin 1972 par William Whitelaw, lors de sa politique de conciliation, mais en janvier 1975, une commission présidée par Lord Gardiner se prononça pour l'abolition de ce statut avançant que les autorités 
pénitentiaires avaient perdu le contrôle des détenus. Le secrétaire d'État travailliste, Merlyn Rees, annonça donc la suppression de ce statut spécial à compter du $1^{\mathrm{er}}$ mars 1976. Voir Report of a Committee to Consider, in the Context of Civil Liberties and Human Rights, Measures to Deal with Terrorism in Northern Ireland (Gardiner Report), London: HMSO, January 1975.

23. Voir les articles des 25 avril, 2 mai, 22 mai et 9 juin 1981.

24. Libération du 23/24 mai 1981.

25. Libération, éditorial du 25/26 juillet 1981.

26. Connus sous le nom de "Diplock courts » : une commission présidée par Lord Diplock conseilla au gouvernement britannique en décembre 1972 d'établir des procès sans jury afin d'éviter toute intimidation des jurés par les organisations paramilitaires. Voir Report of the Commission to Consider Legal Procedures to Deal with Terrorist Activities in Northern Ireland (Diplock Report), London : HMSO.

27. L'Humanité du 22 avril et du 6 mai 1981 par Jean-émile Vidal.

28. L'Humanité du 16 mai, 23 mai, 25 mai 1981, du 6 juillet, du 14 juillet 1981.

29. L'Humanité du 25 mai, p.7, par Jean-émile Vidal.

30. L'Humanité du 6 mai 1981, la Une et p. 7, par Yves Moreau.

31. L'Humanité du 21 avril 1981 par Yves Moreau.

32. L'Humanité du 8 mai, p. 8, par Jean-émile Vidal.

33. Libération a couvert cette campagne électorale en publiant quatre articles sous forme de « chronique du milieu de l'Irlande ", édition des 10 juin, 11 juin, 12 juin et 15 juin 1981, par Sorj Chalandon et L'Humanité la rapporta dans ses éditions des $30 \mathrm{mai}, 1^{\mathrm{er}}$ juin et 15 juin 1981.

34. L'Humanité du 21 avril 1981, la Une et p.10, par Yves Moreau.

35. Respectivement les articles des 23 avril, 25 avril et des 2 mai, 4 mai et 6 mai 1981 par Jean-Émile Vidal et Yves Moreau.

36. L'Humanité des 29 avril et 25 mai 1981, par Jean-Émile Vidal.

37. L'Humanité du 6 mai 1981, la Une et p.7, par Yves Moreau.

38. L'Humanité du 13 mai, la une, par Martine Monod.

39. L'Humanité du 14 mai 1981, la une et p. 6.

40. L'Humanité du 23 mai 1981, p. 9.

41. L'Humanité du 29 mai 1981, la Une et p. 6.

42. Voir les articles du 24, 27, 30 avril 1981 et du 10 et 31 juillet 1981.

43. L'Humanité du 20 mai 1981, la Une et p. 6, par Yves Moreau et du $1^{\text {er }}$ août 1981, la une.

44. Libération du 15 mai 1981.

45. Libération du 9 juillet 1981.

46. Libération, éditorial du 10 août 1981, p. 3.

47. Ibid. Voir également l'article du 21 août 1981, par Sorj Chalandon.

48. L'Humanité du 21 mai et du $1^{\mathrm{er}}$ août 1981, Libération du 18 mai 1981.

49. Respectivement articles du 29 juillet 1981 et du 30 juillet 1981.

50. L'Humanité des 21 avril, 23 avril, 8 mai, 23 mai 1981 entre autres. 
51. Le Comité de Défense des Libertés et des Droits de l'Homme en France et dans le Monde menait également campagne pour la libération d'Annie Maguire et de sa famille, victime d'une erreur judiciaire reconnue en juin 1990 par la justice britannique (Voir les articles de L'Humanité du 28 octobre et 20 décembre 1980).

52. Voir entre autres L'Humanité des 14, 22, 28, 30 avril et 2 juin 1981.

53. Voir également les éditions des 30 avril 1981, 2 mai, 4 juillet, 9, 10 juillet, 12 août, 22, 24,26 et 28 août 1981.

54. L'Humanité des 30 avril 1981, 7 mai, 15 mai, $1^{\mathrm{er}}$ juin, 18, 23, 24, 27, 30 et 31 juillet, 11, 13, 18, 29 août, 4 et 8 septembre 1981.

55. L'Humanité des 14 août, $1^{\mathrm{er}}$ septembre et 7 octobre 1981.

56. L'Humanité du 8 mai par Yves Moreau.

57. Article du 25 avril 1981, la Une et p. 8 par Jean-Émile Vidal.

58. Article du 6 mai 1981 par Yves Moreau. Voir également celui d'André Wurmser du 7 mai 1981.

59. L'Humanité des 22, 24, 25 avril, des 5, 8, 9 et 18 mai 1981.

60. L'Humanité du 14 mai 1981, la Une et p. 6 par Yves Moreau.

61. Libération du 15 mai 1981, par Sorj Chalandon.

62. Article du 25/26 juillet 1981, p. 2.

63. Libération du 10 août 1981, p. 2.

64. Libération, éditorial du 5 octobre 1981. Il est intéressant de noter que L'Humanité n'a pas considéré la cessation de la grève comme une défaite car les prisonniers «n'ont pas donné leur vie en vain » dans la mesure où « dans tous les pays, notamment en France, ils ont sensibilisé l'opinion au drame irlandais », article du 5 octobre 1981 par Yves Moreau.

65. Libération du 3 juillet 1981.

66. Libération du 4 août 1981.

67. Owen Carron était l'agent électoral de Bobby Sands et fut élu député à Westminster à la suite du décès de ce dernier.

68. M. Claude Cheysson, ministre des relations extérieures, a expliqué la position du nouveau gouvernement socialiste : le 15 juillet 1981, il déclare à l'Assemblée nationale que « plus que d'autres, nous devons, nous Français, participer à la recherche d'un règlement, marquer notre profonde sympathie à ce peuple» et exprime son soutien au gouvernement de Dublin, tout en indiquant lors d'une interview à la chaîne britannique ITV le 23 août 1981 que les relations franco-britanniques ne se détérioreraient pas du fait de la grève de la faim.

69. Articles du 21 et 28 octobre 1980 et 13 mai 1981.

70. Articles des 9 juillet, 3 et 10 août 1981.

71. L'Humanité du 6 septembre 1971. 


\section{RÉSUMÉS}

Aucun événement des "Troubles » nord-irlandais n'a provoqué un plus grand intérêt en France que la grève de la faim des prisonniers républicains en 1981, survenue pendant la campagne pour les élections présidentielles en France. Les quotidiens de gauche, Libération et L'Humanité, ont organisé une véritable campagne de soutien des demandes des grévistes de la faim nordirlandais, menée en parallèle d'une virulente campagne contre le colonialisme britannique et contre Margaret Thatcher, jugée responsable du décès des dix prisonniers. Ils ont ainsi saisi l'occasion de démontrer, en période électorale, que la gauche française défendait les droits de l'homme et les valeurs démocratiques françaises.

No other events during the 'Troubles' caused more interest in France than the 1981 Hunger Strike and Bobby Sands' death, which occurred during the French Presidential campaign and which were appropriated by the left-wing paper Libération and the pro-Communist L'Humanité to prove that the French Left was able to defend human rights and French democratic values. They campaigned for the hunger strikers' demands and against both British colonialism and Margaret Thatcher, held personally responsible for the ten hunger strikers' deaths.

\section{INDEX}

Keywords : Northern Ireland - conflict, Northern Ireland - hunger strikes (1981), Franco-Irish relations, press, history of representations, human rights

Mots-clés : Irlande du Nord - conflit, Irlande du Nord - grèves de la faim (1981), relations francoirlandaises, presse, histoire des représentations, droits humains

\section{AUTEUR}

\section{KARINE DESLANDES}

University of Buckingham 